\title{
Synchrotron Phase Space Injection Into LEP
}

\author{
P.Collier, CERN, 1211-Geneva, Switzerland
}

\begin{abstract}
Although LEP was designed to allow both betatron and synchrotron injection, only betatron accumulation has been used up to now. During 1994 tests were made of injection in synchrotron phase space. Results are presented from these machine studies which show that for both 4 bunch and 8 bunch pretzel injection, this form of injection is a more efficient way of accumulating LEP beams. The use of synchrotron injection has several advantages, including faster damping and less sensitivity to injection errors. In addition, the flat trajectories in the straight sections, which are a feature of this type of injection, can lead to lower radiation doses for the LEP experiments and open the possibility of injection into tuned optics. Tests with high phase advance lattices also indicate that high efficiency injection is easier to achieve with synchrotron injection. For the final running period of LEP in 1994, synchrotron injection was used for normal operations and will be the preferred method used in 1995.
\end{abstract}

\section{INTRODUCTION}

During the design phase of LEP, the possibility of using synchrotron injection was discussed [1]. In the final LEP design, the placement of the injection elements was made such that synchrotron injection was possible. Since the LEP start-up in 1989, however, no attempts were made to exploit this possibility. Instead, use has been made exclusively of betatron phase space injection.

For each particle type the injection elements of LEP consist of a magnetic septum, which acts on the injected beam together with three kicker magnets, which move the circulating beam close to the septum during injection. The injection points are placed in the arcs, symmetrically around one of the LEP straight sections. Injection is in the horizontal plane on the inside of the main ring.

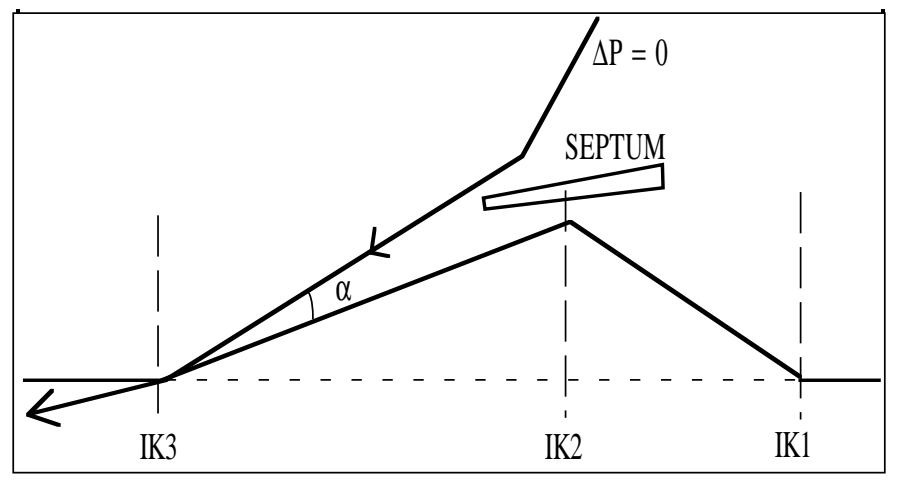

Figure 1: Schematic Diagram Representing Betatron Injection. The angle $\alpha$ is minimized for optimum injection
In betatron injection, the circulating beam is brought as close as possible to the septum with the kickers and the injected beam is steered to minimize the angle between the injected and circulating beams. Due to the finite width of the septum, this angle, $\alpha$, can never be zero. The injected beam then performs betatron oscillations around the closed orbit of the circulating beam, eventually damping into it. The transverse damping time at injection $(20 \mathrm{GeV})$ in LEP is about 6000 turns. This form of injection is shown schematically in figure 1 .

Injection in synchrotron phase space requires that there is a non-zero dispersion, in the plane of the injection, at the injection point. The same use is made of a kicker bump to bring the circulating beam close to the septum. In this case, however, the beam is injected with an offset in energy. As the injection lines to LEP are on the inside of the ring, this offset must be negative. To optimize the injection process the distance between the injected and circulating beam at the injection point is matched to the energy offset of the injected beam, using the relation :

$$
x=D_{x} \cdot \Delta P / P
$$

By adjusting the septum the beam can be injected parallel to the circulating beam and will follow the natural closed orbit that a circulating particle, having the given energy offset, would follow [2]. This is illustrated schematically in figure 2.

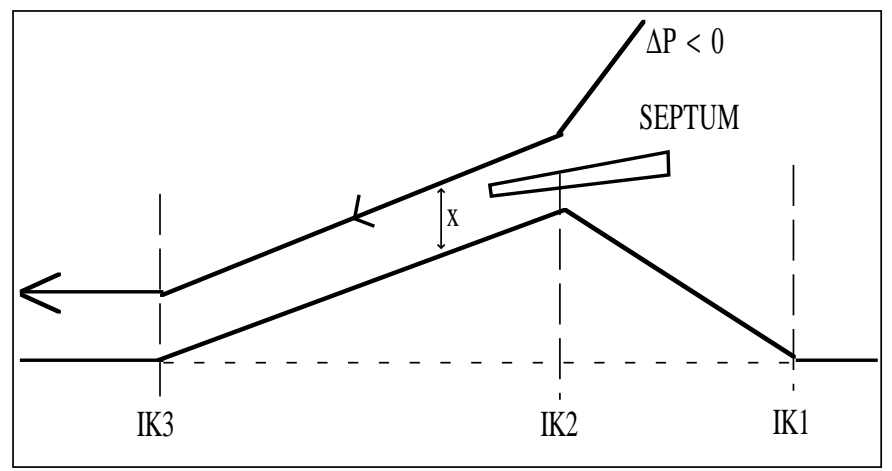

Figure 2: Schematic Diagram Representing Synchrotron Injection with $\mathrm{x}=\mathrm{D}_{\mathrm{x}} \cdot \Delta \mathrm{P} / \mathrm{P}$.

In the case of synchrotron injection the angle between the injected and circulating beams can be adjusted to be zero and hence the injected beam will not perform betatron oscillations about the closed orbit. Instead, once injected, the beam oscillates in the longitudinal plane (energy oscillations) at the synchrotron tune (Qs) and slowly damps into the circulating beam. 


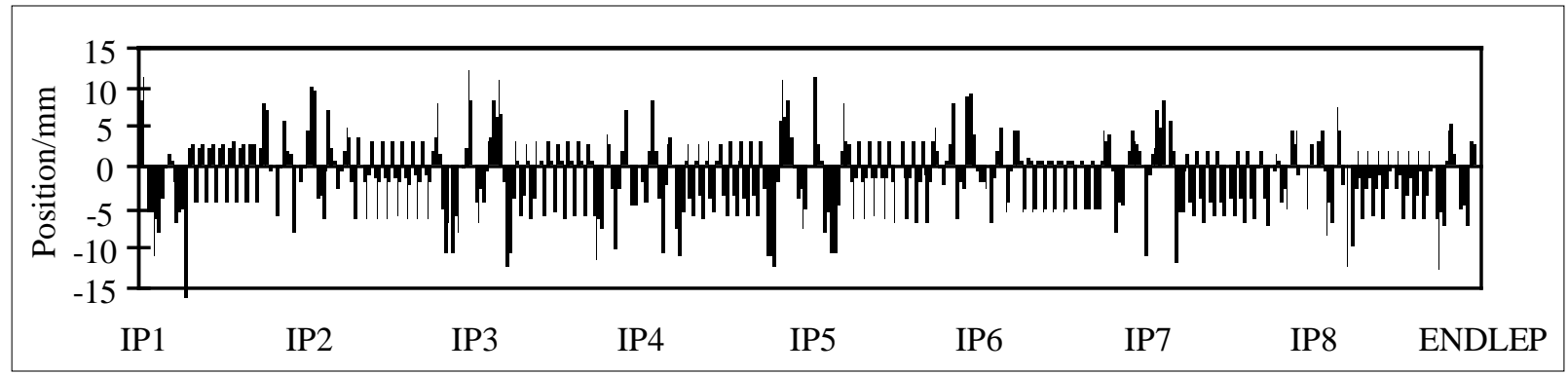

Figure 3: Optimized Horizontal First Turn Trajectory for Betatron Injection of Positrons into LEP.

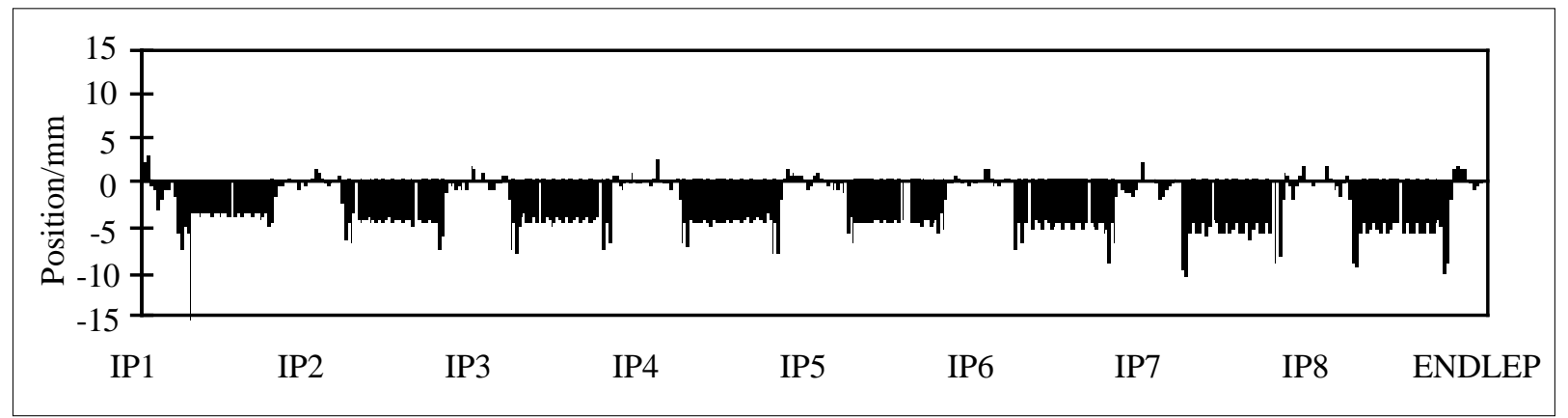

Figure 4 : Optimized Horizontal First Turn Trajectory for Synchrotron Injection of Positrons with $\Delta \mathrm{P} / \mathrm{P}$ at $-0.6 \%$

\section{INJECTION}

In the longitudinal plane the damping time is a factor of 2 lower than in the transverse plane. Therefore, the synchrotron injection oscillations damp out after about 3000 turns in LEP. The injected beam sees non-linear RF fields and different particles thus have different values of $Q_{s}$. After a number of energy oscillations the injected beam tends to smear out in longitudinal phase space. This is known as filamentation [1] and is considered to be advantageous as it results in a rapid suppression of any injection oscillation signal.

The main advantage of synchrotron injection is that there are no injection oscillations in the straight sections. This is because the horizontal dispersion is zero in these regions. This allows cleaner injection conditions for the LEP experiments. Figures 3 and 4 compare and contrast the optimized trajectories measured with betatron and synchrotron injection respectively.

Other advantages have been found for synchrotron injection over injection in betatron phase space. Notably, the larger dynamic acceptance in the longitudinal plane allows a very high injection efficiency. Injection into an empty machine routinely produces $100 \%$ injection efficiency. In addition the reduced (or zero) betatron oscillations at injection allow the LEP transverse feedback system to operate more easily.

For comparison purposes the data of figures 3 and 4 are plotted on the same scale. In both cases the injection elements were optimized to minimize the betatron oscillations about the closed orbit. In the case of betatron injection this results in an oscillation of $+/-4 \mathrm{~mm}$ in the arcs, but with peaks of $12 \mathrm{~mm}$ within the straight sections of
LEP. In the case of synchrotron injection the betatron oscillations are completely suppressed and the trajectory follows the dispersion function of the machine.

The data of figure 4 was taken with a $-0.6 \%$ energy offset for the injected beam. This offset can be changed. In order to keep $\mathrm{x}$ constant in equation (1), increasing the energy offset allows the kicker bump to be decreased. The limit on increasing the energy offset comes from the variation of the betatron tunes with momentum. For the optics in use for LEP the limit is at about $-2 \%$ where the horizontal tune drops towards the integer. In figure 5 the injection efficiency into an empty machine is plotted as a function of the energy offset of the injected beam. The efficiency here is defined as the proportion of the beam surviving after $400 \mathrm{~ms}$.

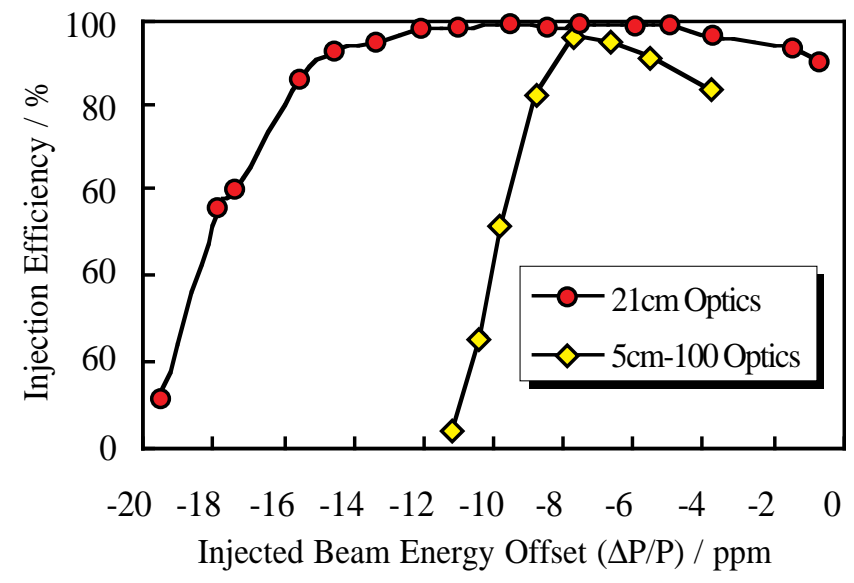

Figure 5 : Variation of the Injection Efficiency into an Empty Machine as a Function of $\Delta \mathrm{P} / \mathrm{P}$ for $21 \mathrm{~cm}$ and $5 \mathrm{~cm}$ Optics. 
Two curves are shown in figure 5 , that for the normal detuned injection optics, where $\beta_{\mathrm{v}}^{*}$ is $21 \mathrm{~cm}$ and for the physics optics, where $\beta^{*}{ }_{\mathrm{v}}$ is reduced to $5 \mathrm{~cm}$. For the standard injection optics energy, offsets up to $1.5 \%$ are possible with good injection efficiencies. Beam can be injected up to $2 \%$ off in energy, but the efficiency drops rather steeply. For the physics optics the latitude for changing the energy offset is rather smaller. In this case attempts to inject in betatron phase space were not successful.

The number and arrangement of bunches in LEP has become a variable which changes at regular intervals. Tests of injection onto pretzel orbits ( 8 bunches per beam) and into bunch trains (with 4 trains of 4 bunches) have been made and in each case the performance of injection in synchrotron phase space proved superior to betatron injection under similar conditions. Attempts have been made to inject using synchrotron injection into other optics configurations. For LEP 2 operation above the $\mathrm{W}$ pair threshold, it is desirable to run LEP with a low-emittance lattice. Two optics are under study, one with $108^{\circ}$ and the other with $135^{\circ}$ horizontal phase advance per cell. In both cases the injection efficiency was much higher when synchrotron injection was used. For the 108 lattice, for example, injection efficiency into an empty machine was above $90 \%$. With betatron injection the efficiency was never higher than about $40 \%$.

\section{ACCUMULATION}

Figure 6 shows the variation of the injection efficiency with accumulated bunch current, for the case of betatron injection and synchrotron injection, for the same optics configuration. Also shown is the curve for injection into physics optics using synchrotron injection. In this case accumulation was not possible with betatron injection.

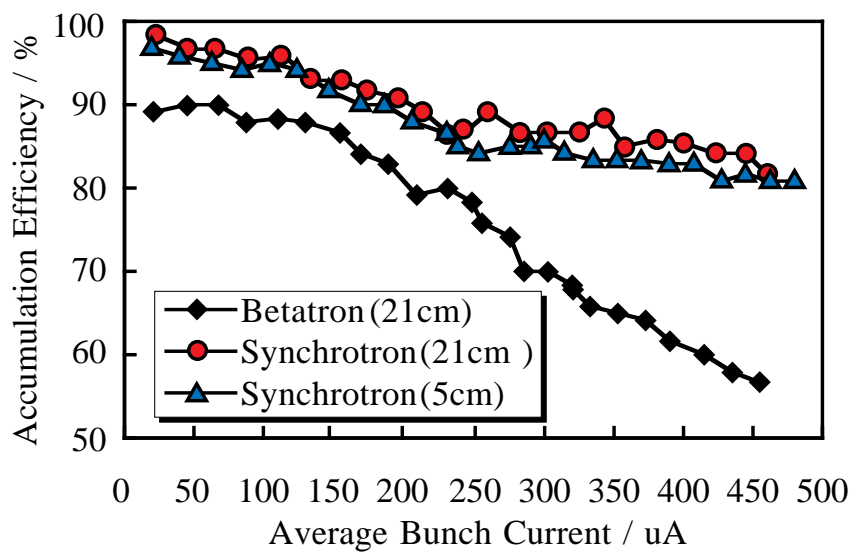

Figure 6: Accumulation Efficiency vs. Accumulated Bunch Current for Betatron and Synchrotron Injection. Note the Suppressed Zero Scale.

A reduction in the efficiency with accumulated bunch current is normal in LEP. The emittance of the beam increases with accumulated current and more losses against the septum are to be expected. In addition, the lifetime of the accumulated beam tends to be lower at high bunch currents.
In the case of synchrotron injection this reduction in efficiency with accumulated current is much smaller; and with $500 \mu \mathrm{A}$ per bunch (nominal bunch currents for LEP), it is still around $85 \%$. Little, or no, reduction in accumulation efficiency can be seen between the tuned and de-tuned optics.

\section{RAPID SYNCHROTRON ACCUMULATION}

LEP plans to operate in 1995 with 4 trains of bunches in each beam. In previous years LEP operated a pretzel scheme and the LEP injectors were modified to allow operation with 8 bunches. The injector chain of accelerators would like to keep the 8 bunch mode operational [3], while still allowing injection into LEP bunch trains. This requires that two bunches from the injectors can be injected into a single LEP bunch within a very short time (a few LEP turns).

Synchrotron injection opens up the possibility of doing this. The method relies on the fact that the synchrotron tune is very low (in LEP Qs is around 0.1). One energy oscillation period is therefore about 10 turns. By waiting some multiple of half a synchrotron period $(5,15,25 \ldots$ turns $)$ between injections into the same bunch, the second injection can be made without disturbing the first. This is because the first injected beam will have too much energy and hence be further away from the septum. The method seems practicable [4] and the necessary modifications to the harmonic numbers of the injector accelerators have been made. It will be tried during the start-up of LEP this year.

\section{CONCLUSIONS}

Synchrotron injection has been found to work well for all machine conditions tested. It is now the preferred operational method of injection into LEP. In general, higher injection efficiencies are normal with synchrotron injection and lower experimental radiation doses are observed. As the trajectory is essentially flat in the straight sections of the machine (the most delicate areas), the performance of the injection process is less sensitive to injection position and angle errors. With no injection oscillations in the straight sections, injection into physics optics has proved to be a practical option. This would avoid the need for a beta-squeeze at high energy and reduce the time needed to set LEP up for physics.

\section{REFERENCES}

[1] S. Myers, "A Possible New Injection and Accumulation Scheme for LEP”, LEP Note 334, 1981.

[2] P. Collier, "Synchrotron Phase Space Injection into LEP", CERN, SL-MD Note 152, 1994.

[3] P. Collier, "Injectors, Status and Options for 1995", Proceedings, Fifth LEP Performance Workshop, Chamonix, France, 1995.

[4] P. Collier, 'Synchrotron Injection', Proceedings, Fifth LEP Performance Workshop, Chamonix, France, 1995. 